\title{
Graph-Based Simulation for Cyber-Physical Attacks on Smart Buildings
}

\author{
Rahul Agarwal \\ Thesis submitted to the Faculty of the \\ Virginia Polytechnic Institute and State University \\ in partial fulfillment of the requirements for the degree of \\ Master of Science \\ in \\ Computer Science and Application
}

Na Meng, Chair

Xinghua Gao

Edward Fox

Anuj Karpatne

May 10, 2021

Blacksburg, Virginia

Keywords: cyber-physical attack, smart building, graph-theory, agent-based simulation Copyright 2021, Rahul Agarwal 


\title{
Graph-Based Simulation for Cyber-Physical Attacks on Smart Buildings
}

\author{
Rahul Agarwal
}

(ABSTRACT)

As buildings evolve towards the envisioned smart building paradigm, smart buildings' cybersecurity issues and physical security issues are mingling. Although research studies have been conducted to detect and prevent physical (or cyber) intrusions to smart building systems (SBS), it is still unknown (1) how one type of intrusion facilitates the other, and (2) how such synergic attacks compromise the security protection of whole systems. To investigate both research questions, the author proposes a graph-based testbed to simulate cyber-physical attacks on smart buildings. The testbed models both cyber and physical accesses of a smart building in an integrated graph, and simulates diverse cyber-physical attacks to assess their synergic impacts on the building and its systems. In this thesis, the author presents the testbed design and the developed prototype, SHS Im. An experiment is conducted to simulate attacks on multiple smart home designs and to demonstrate the functions and feasibility of the proposed simulation system. 


\section{Graph-Based Simulation for Cyber-Physical Attacks on Smart Buildings}

Rahul Agarwal

(GENERAL AUDIENCE ABSTRACT)

A smart home/building is a residence containing multiple connected devices which enable remote monitoring, automation, and management of appliances and systems, such as lighting, heating, entertainment, etc. Since the early 2000s, this concept of a smart home has become quite popular due to rapid technological improvement. However, it brings with it a lot of security issues. Typically, security issues related to smart homes can be classified into two types - (1) cybersecurity and (2) physical security. The cyberattack involves hacking into a network to gain remote access to a system. The physical attack deals with unauthorized access to spaces within a building by damaging or tampering with access control. So far the two kinds of attacks on smart homes have been studied independently. However, it is still unknown (1) how one type of attack facilitates the other, and (2) how the combination of two kinds of attacks compromises the security of the whole smart home system. Thus, to investigate both research questions, we propose a graph-based approach to simulate cyberphysical attacks on smart homes/buildings. During the process, we model the smart home layout into an integrated graph and apply various cyber-physical attacks to assess the security of the smart building. In this thesis, I present the design and implementation of our tool, SHSım. Using SHSım we perform various experiments to mimic attacks on multiple smart home designs. Our experiments suggest that some current smart home designs are vulnerable to cyber-physical attacks. 


\section{Dedication}

To my mother, Nisha Agarwal.

For all the love and support

she has provided me as a single parent. 


\section{Acknowledgments}

First and foremost, I would like to thank almighty God for the blessings and for keeping me healthy while I was busy with the research work.

I am thankful to Virginia Tech for providing me with opportunities and a haven in this foreign land. Research is challenging and requires a lot of patience to get the results. I remember my first research idea, for which I spent a semester, didn't work out. But, thanks to my advisor Professor Na Meng for believing in me with another project. Prof. Na Meng has been a great mentor and constant support on this journey. I am indebted to Professor Xinghua Gao for providing me with the domain knowledge and support throughout this thesis. I would also like to thank Professor Edward Fox and Professor Anuj Karpatne for

serving on my committee. I am thankful to Yuqing Liu for kickstarting the project and providing all the resources and knowledge to take it forward.

Finally, I am immensely grateful to my mother for her love, prayers, and sacrifices for educating me and preparing me for the future. 


\section{Contents}

List of Figures $\quad$ viii

List of Tables $\quad$ ix

1 Introduction $\quad 1$

2 Literature Review $\quad 5$

2.1 Cyber Attacks on Smart-home Ecosystem . . . . . . . . . . . . . . 5

2.2 Physical Attack Simulation on Smart-home/Smart buildings . . . . . . . . 6

2.3 Smart-home System Design . . . . . . . . . . . . . . . . 7

3 Methodology $\quad 9$

3.1 User Input . . . . . . . . . . . . . . . . . . . . . 10

3.2 Graph Generation . . . . . . . . . . . . . . . . . 12

3.3 Path Enumeration . . . . . . . . . . . . . . . . . . 14

3.4 Vulnerability Detection . . . . . . . . . . . . . . . . 15

3.5 Detected Attacks Visualization . . . . . . . . . . . . . . . . 17

4 Results $\quad 19$

4.1 Smart home layout $1 \ldots \ldots \ldots$. . . . . . . . . . . . . . . . . . . . . . 
4.1.1 Experiment 1a: Space monitored vs not monitored . . . . . . . . 19

4.1.2 Experiment 1b: Smart Speaker visibility . . . . . . . . . . . 21

4.2 Smart home layout $2 \ldots \ldots$. . . . . . . . . . . . . . . . . 21

4.2.1 Experiment 2: All spaces monitored . . . . . . . . . . . 23

4.3 Smart home layout $3 \ldots \ldots \ldots$. . . . . . . . . . . . . . . . 25

4.3.1 Experiment 3: No occupants . . . . . . . . . . . . 27

5 Discussion $\quad 30$

6 Conclusions $\quad 32$

$\begin{array}{ll}\text { Bibliography } & 33\end{array}$ 


\section{List of Figures}

3.1 Architecture of SHSIM . . . . . . . . . . . . . . . . . . . . 9

3.2 SHSim interface to provide information about the smart home layout . . . . 11

3.3 An example input graph of a smart home system . . . . . . . . . . . . 13

3.4 Light command attack on Smart home layout $1 \ldots$. . . . . . . . . . 18

4.1 Smart home layout $1 \ldots \ldots$. . . . . . . . . . . . . . . 20

4.2 Floor plan of Smart home layout $2[1] \ldots \ldots$. . . . . . . . . . . . 22

4.3 Internet setting in Smart home layout $2 \ldots \ldots . \ldots . . \ldots 23$

4.4 Security system setting of Smart home layout $2 \ldots$. . . . . . . . . . . 24

4.5 Automation system setting of Smart home layout $2 \ldots$. . . . . . . . . 24

4.6 An example DDoS attack on smart security system of Smart home layout 2 . 25

4.7 Light command attack on Smart home layout $2 \ldots$. . . . . . . . . . 26

4.8 Smart home layout $3 \ldots \ldots$. . . . . . . . . . . . . . . 27

4.9 Concealed jamming attack on Smart home layout $3 \ldots$. . . . . . . . 29 


\section{List of Tables}

3.1 All attacks modeled in SHSim . . . . . . . . . . . . . . . . 17 


\section{Chapter 1}

\section{Introduction}

Smart home automation is gaining popularity due to the convenience and facilities it provides to homeowners. For instance, owners can control and monitor indoor environments using their smartphones. Smart home devices (e.g, voice-controlled home assistants, smart light bulbs, smart switches, smart thermostats, smart door locks, etc.) connect into a common network that can be independently and remotely monitored. Owners of the devices enjoy the convenience of access to information through voice commands, remote controls, and enhanced home security by the power of monitoring cameras and sensors. To provide such convenience, the devices store or transmit sensitive information, such as account information and live footage, which makes them cyber targets for attackers. On the other hand, smart home devices interact with the physical space as they can alter the lighting, configure AC settings, monitor the space, unlock doors, etc. Breaching of physical space can lead to physical access to the devices, and breaching of some devices that can render the physical space insecure.

Today, many smart home systems are powered by state-of-the-art technology and provide great compatibility with third-party devices. For instance, Google's Home Assistant or Amazon's Echo can be used both as smart speakers as well as to connect with other smart home devices such as Nest Smart Lock or Philips smart lighting. The integration provides a much smoother smart home experience to the users as they can choose from numerous product configurations from different providers. However, studies show that certain product 
configurations are not always secure. For instance, Huraj et al. [12], in their research on Denial of Service (DDoS) attack, found out that when Fibaro Home Center 3 is being flooded with HTTP GET requests, the connection between Amazon Alexa mobile app and the wall plug sensor gets disrupted. This shows that there exist security loopholes when trying to integrate smart home devices from different vendors.

In recent years, smart home manufacturers are investing extensively in cybersecurity. However, newer and more complex cyberattacks such as Light command attack [26] keep getting developed, and can breach current smart home systems. On the other hand, physical security is relatively well-studied. However, physical security cannot offer sufficient protection for the average homeowner. To better study the security in the smart home devices and the space they reside, we must consider the two securities and their interactions, known as cyber-physical security.

Unlike most cybersecurity threats, cyber-physical threats are of increasing concern. In 2019, Gartner predicted that the financial impact of Cyber-Physical Systems (CPS) attacks resulting in fatal casualties will reach over $\$ 50$ billion by 2023. According to the article [29], CPS "attacks can also entail a loss of customers, intellectual property theft, operational shutdowns or degraded equipment performance". Currently, there is no systematic approach to measure such attacks. To tackle this problem, we believe that the simulation of the entire system is desirable. Also, there isn't a tool which can estimate the cyber-physical security of a smart home environment. Thus, in this thesis we present a simulation framework to investigate the security of a smart home environment and provide some recommendations on which smart home solution is more secure. With our tool, SHSım, researchers and customers can compare security levels of different smart home solutions. By analyzing the robustness of a given smart home solution, customers can make better decisions regarding which product they should purchase. Lastly, our study can help and motivate smart-home vendors to 
design a more secure suite of products.

SHSim includes a web interface to take user input of their smart home layout, a simulation engine that generates all possible attack paths, and a graph-based visualization of different attack models. In our solution, we first map the smart home layout to an integrated graph (consisting of nodes and edges). Then, we traverse the graph with different starting points to generate all possible paths. We check the generated paths against various attack models and output all successful attack paths. In our experiments, we tested the vulnerability of two smart home ecosystems along with different configurations to check the robustness of the simulation engine. We found out that various cyber-physical attacks can be employed to breach the smart home ecosystem. The simulation engine exploits the vulnerability of popular communication protocols to generate all possible attack paths using which an attacker can gain access to critical smart home devices. To allow other researchers to use our tool, we have made SHSıм publicly available at https://github.com/rahulaVT/SIM_app.

Here we define some of terminologies being used throughout the thesis:

1. Smart home system is a suite of smart home devices used for home automation or home security purpose. Some of the popular smart home automation systems include Google Nest and Amazon Alexa. ADT and Simplysafe are popular choices for smart home security systems.

2. Cyber attack is a network-based attack launched by cybercriminals/hackers to maliciously gain network access, steal user data, or breach access points.

3. Physical attack means breaking into a home by damaging physical infrastructure (doors, windows, etc.) to gain physical access to space or devices.

4. Cyber-Physical attack is a security breach in cyberspace, which adversely affects physical space. 
5. Simulation engine is a program that allows the user to observe an operation through simulation without actually performing that operation.

The rest of the thesis is organized as follows. In Chapter 2 we shed light on some previous work that has been done in this domain. In Chapter 3 we comprehensively describe our research methodology. The results and analysis are explained in Chapter 4, and in Chapter 5 we have included a discussion on the threats to validity and possible solutions. Finally, in Chapter 6 we present our conclusion. 


\section{Chapter 2}

\section{Literature Review}

While performing this study, the author primarily researched about three research areas for our literature review: (1) cyber attacks on smart-home ecosystem (Section 2.1), (2) physical attack simulation on smart-home/smart buildings (Section 2.2), and (3) smart-home system design (Section 2.3).

\subsection{Cyber Attacks on Smart-home Ecosystem}

The cybersecurity of smart home devices is being studied extensively as new cyberattacks keep getting implemented. In order to simulate different attack models, we did a comprehensive literature review of cyber and physical attacks on IoT devices. Distributed Denial of Service (DDoS) [36] is one of the most common attacks on IoT networks. It is an attempt to flood the server with internet traffic to shut down the service either completely or partially. The experiments conducted by Huraj et al. [12] show how an attacker from outside can disturb the normal operation of smart home devices when they become victim of a DDoS attack. Gómez et al. [7] in their research work expose the vulnerability of ZigBee devices for wormhole attacks and packet injection. According to Gómez et al., a successful wormhole attack can "impact the confidentiality, availability, and integrity of the data in the Wireless Sensor Network (WSN), which could affect part or all of the network". A Jamming attack is another popular cyberattack where an adversary pretends to be a legitimate 
WIFI device that prevents/delays the sensor's communication to the gateway. Chi et al. [3] present a variant of jamming attack called concealed jamming attack that targets Zigbee devices, preventing them from receiving Zigbee packets that may contain important information. Researchers have found many other cyber attacks which target the vulnerability in communication protocols to leak sensitive information from devices [6, 28].

\subsection{Physical Attack Simulation on Smart-home/Smart buildings}

Ciholas et al. [4] performed a systematic literature review of the security of Smart Buildings (SBs) wherein they investigated 90 relevant research papers. Ciholas et al. found out that "very few papers consider the issue about physical attacks on sensors, actuators, and controllers in SB". In [9], the authors identified potential physical attacks such as vandalism, burglary, and theft of SB devices. In [18], the researchers exposed the vulnerability of KNXbased devices present at the field layer. The researchers could connect to the KNX network from a simple connected light switch and gain full control of the building. Heartfield et al. [10] devised a novel taxonomy of 25 different smart home attacks, classifying attack vectors and their impact on physical, domestic, and emotional well-being. Building Information Model (BIM) can be used to generate graphs, reflecting spatial relations between spaces

within a building [20, 25]. Porter et al. [20] proposed a way to use the generated graph to simulate a physical attack on the building to see which rooms and obstacles (windows, doors, walls) are the most vulnerable. Skandhakumar et al. [25] utilized the graph representation of BIM for indoor navigation applications. Regardless of the novelty of this approach, it does not take into account the concept of smart devices that can be affected by people with access to the building spaces. Our research work takes inspiration from the work done by Porter 
et al. and Skandhakumar et al. to model smart home systems using graphs. However, we simulate both cyber and physical attacks on the smart home system to identify potential attack paths.

\subsection{Smart-home System Design}

Designing a Smart Home requires planning. There are a variety of customization options available to create a smart home: audio, lighting, HVAC, and security solutions. The homeowner needs to consider how the house will function as a whole [15], the level of automation required, strategic placement of devices, and cost to build the system. Robles et al. [23] reviewed a few smart home system designs and provide ten guidelines to help people design their own smart homes. Designing a sophisticated smart home may require professional assistance that can be costly. Hu et al. [11] assess the usability of self-installation kits intended for the senior population. Their study indicated that the installation of a few devices from a Smart Home in a Box (SHiB) system has a high success rate even for individuals with no engineering background. Yamazaki et al. [35] introduced the concept of "The Ubiquitous Home" which consists of several context-aware services and a robot acting as an interface for the residents.

Smart home technology promises tremendous benefits for the elderly and people with disabilities. We found a few research studies related to the smart home design catering to elderly people $[5,14,21]$. Majumder et al. [16] presented a comprehensive review on the state-of-the-art research and development in smart home based remote healthcare technologies. According to them, "Smart homes can enable continuous and remote monitoring of elderly health and well-being at a low cost".

Prior research work on smart home system design is geared towards specific automation 
applications. However, in our research work, we focus on the security aspect of smart home systems. Although physical and cybersecurity of smart home environments have been studied independently, there is no tool that can simulate cyber-physical attack together. This research presents a graph-based simulation technique to model both physical and cyberattacks of a smart home environment. 


\section{Chapter 3}

\section{Methodology}

Figure 3.1 presents the system architecture of SHSıм. Our approach primarily consists of five steps. In Step 1 we expect the user to provide the information about their smart-home architecture using the web interface (Section 3.1). In Steps 2-4 we use automated scripts written in Python 3 to identify vulnerable cyber-physical attacks (Section 3.2 - Section 3.4). Finally, in Step 5 we use D3.js [2] to provide interactive graphical visualization of detected vulnerable attacks (Section 3.5).

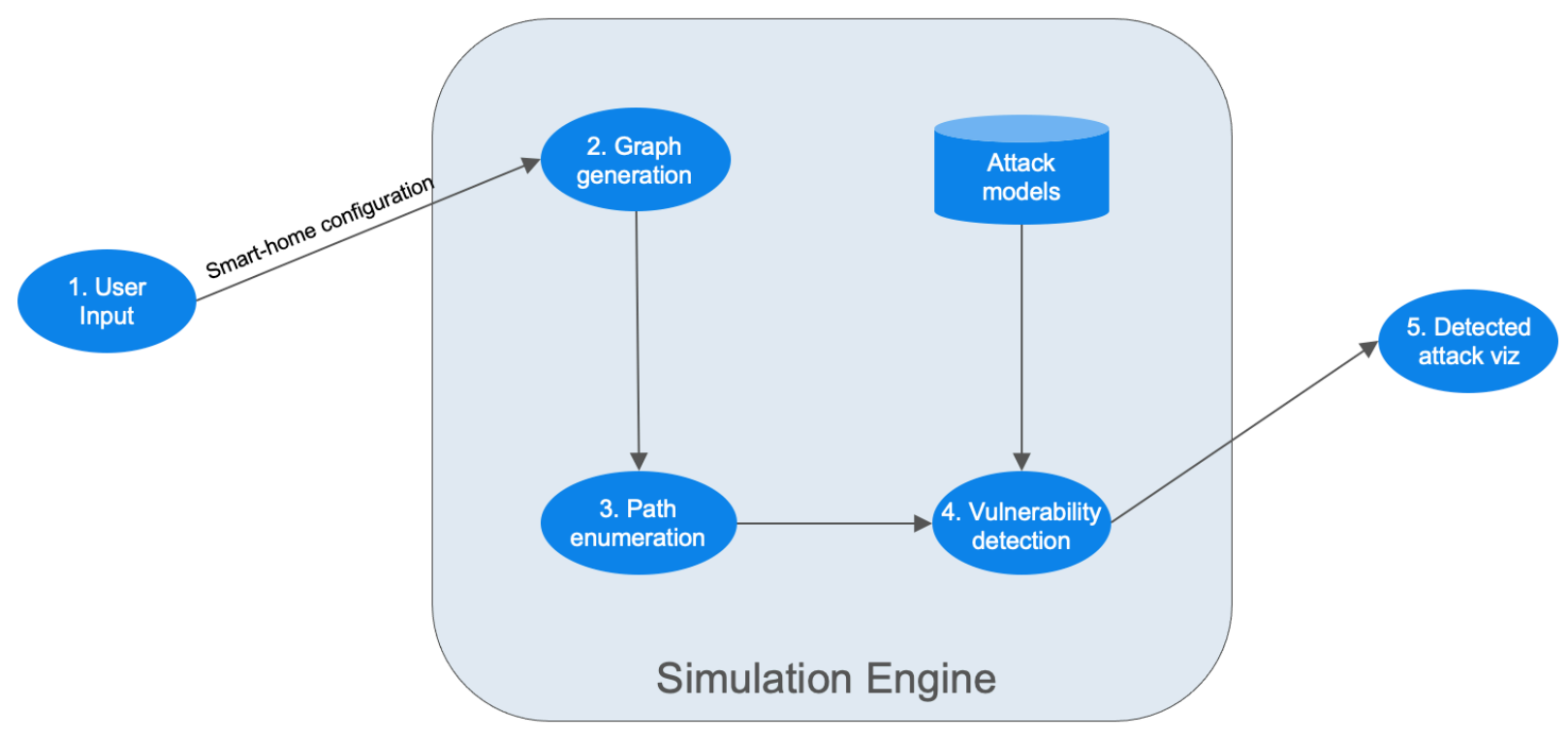

Figure 3.1: Architecture of SHSIm 


\subsection{User Input}

We have used Node.Js [19] \& ReactJS [22] for front-end and Python \& Flask [8] for running the back-end of our web application. Node.js is an open-source, cross-platform runtime environment used for developing fast, scalable server-side and network applications. Developers prefer to use ReactJS in combination with Node.js to build reusable user interface (UI) components because React uses a JavaScript virtual DOM, which is faster than an ordinary DOM [13].

The first step to running the simulation is to provide the smart-home configuration which includes details about the floor plan and the placement of smart home devices within the home. Figure 3.2 describes the user interface of SHSIm that includes four paper components [17] to take user input.

1. Add Spaces component consists of textfields and checkboxes to define space configuration.

2. Add Devices component consists of a textfield and drop-down selections to create and place devices within the smart home.

3. Create Physical Connection component consists of drop-down selections and a checkbox to define connections between spaces.

4. Create Cyber Connection component consists of drop-down selections and a checkbox to define connections between devices.

The components also contain a list-view of items being added with the option to edit or delete items as required. The provided information gets saved as a JSON file by clicking the submit button. We have given the flexibility to load an existing JSON file, make changes, and then run the simulation. 

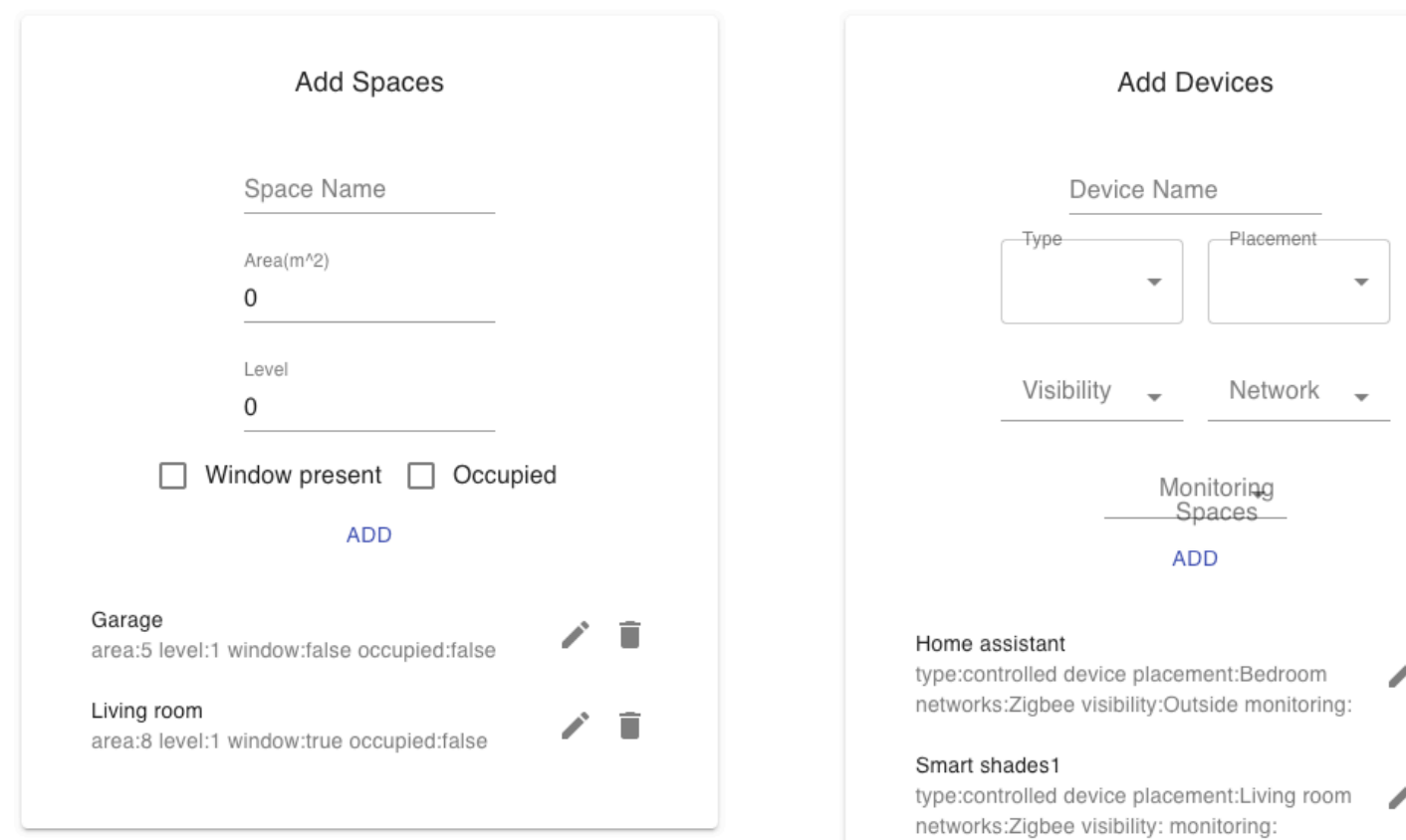

Home assistant

type:controlled device placement:Bedroom

networks:Zigbee visibility:Outside monitoring:

Smart shades 1

type:controlled device placement:Living room

networks:Zigbee visibility: monitoring:

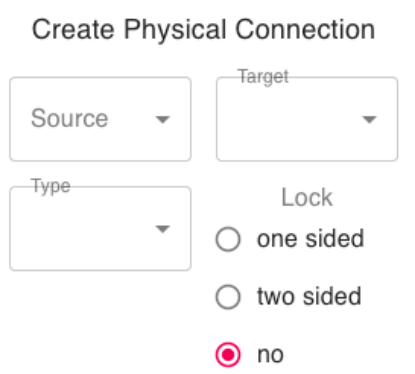

ADD

pc_1

sources:Living room targets:Bedroom type:door lock:no

pc_2

sources:Living room targets:Storage room type:dgei lock:no

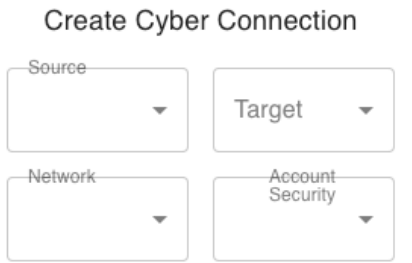

Network Security

weak

medium

strong

ADD

cc_1

sources:Home assistant targets:Smart shades2

networks:Zigbee securityTypes:Amazon

securityLevel:weak

cc_2

sources:Home assistant 2 targets:Smart

shades1:Smart light networks:Zigbee

securityTypes:Amazon securityLevel:medium

SUBMIT RUN SIMULATION LOAD DATA

Figure 3.2: SHSim interface to provide information about the smart home layout 


\subsection{Graph Generation}

Graphs have been widely used in building network topology, social networks, biological networks, operational research, etc. [30]. A graph consists of vertices that are connected by edges. A graph may be undirected, meaning that there is no distinction between the two vertices associated with each edge, or its edges may be directed from one vertex to another. We have used graphs to model various smart home configurations, combining with agent-based simulation to analyze the cyber-physical security of smart homes.

In order to model the smart-home system, we must define nodes and edges. We created SpaceNode to define the physical spaces such as hallway, kitchen, bedrooms, etc., and DeviceNode to define the smart home devices. Each SpaceNode holds a set of attributes such as area, floor level, window present (True/False), and occupied (True/False). DeviceNode has attributes such as type, placement, network, visibility, monitoring, etc. The edges connecting the nodes are of three types: physical connection, cyber connection, and containment connection. The physical connection is represented as a solid black line with a stroke-width of 1.5 as shown in Figure 3.3. If there is a physical edge connecting two spaces, it means that the attacker can access and move between the spaces. However, if space is occupied, there wouldn't be a physical edge connecting that space, and thus, the attacker is prohibited from entering the occupied space. Similarly, if space is being monitored by a smart device such as a smart camera or a glass break sensor, the attacker would not try to physically enter that monitored space.

The cyber connection shows connectivity between smart home devices. It has been represented as dotted lines in Figure 3.3. For our study, we have focused on devices with wireless communication since most smart home devices are designed to be wireless. Generally, the smart home environment consists of two kinds of devices: low-powered IoT devices/sensors 
(e.g., smart thermostat, smart lock, etc.) which run on a lithium-ion battery and have a shorter range of communication; and high-powered IoT devices (e.g., smart home assistants, smart light, smart camera, etc.) which need an external power supply and can transmit over a longer range. The low-powered sensors are paired with a gateway and they follow a certain communication protocol (e.g., Zigbee [24], Z-Wave [32], WIFI, Bluetooth, etc.) to talk to each other. These communication protocols are designed to ensure reliable and secure transmission of IoT sensor data at the edge.

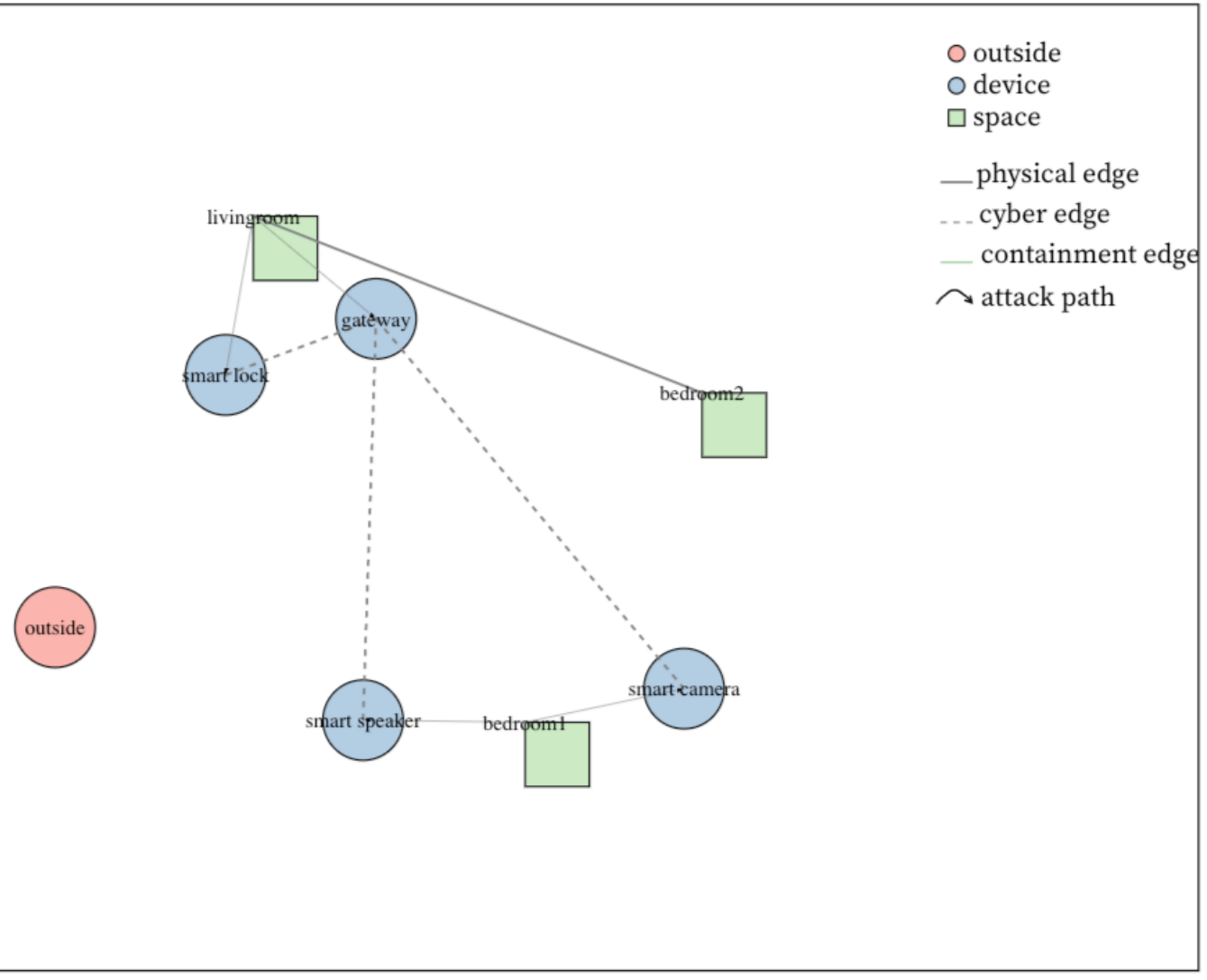

Figure 3.3: An example input graph of a smart home system

The placement of devices within spaces is defined by a containment edge which is represented as a green solid line. There can be zero or more devices present in a given space. The containment edge is useful in scenarios where we want to model a cyber-physical attack. For instance, an attacker can break into space (if it's not being monitored or occupied) and gain 
physical access to all the devices present in that space. Also, certain attacks require direct visibility of devices which may be possible by breaking into a space and accessing vulnerable

devices to perform a cyber attack. Laser-based voice command injection [26] works when the attacker has direct visibility of voice assistant-based smart devices.

\subsection{Path Enumeration}

Once the graph is generated, different simulations can be performed on it, either locally or remotely in the cloud of the service provider. The graph-based modeling of the system reflects what the attacker can observe. The simulation follows the same logic. Our simulation engine works by taking into account certain assumptions about the attacker's capabilities.

- The attacker must have a start position. For smart home simulation, a simple example can be that the attacker is located outside (represented as a orange circle in Figure 3.3) with access to the internet.

- The attacker must also have access to some nodes to initiate attacks.

- In order to initiate a cyber attack, we assume that the attacker can detect some wireless signals.

- To simulate the attack method mentioned in [26], the visibility of the microphone must be modeled so the attacker can "see" the device and establish such an attack.

- In order to initiate a physical attack, we assume that the attacker has tools to detect whether space is being monitored or not.

We have used the Depth First Search (DFS) algorithm to traverse the generated graph and enumerate all possible paths between any two given nodes in the smart home system. We 
built a function called "generatePaths" which takes the start and end node as input parameters and returns all paths between the two nodes. The function "generatePaths" yields valid paths based on the integrated graph which encapsulates the access control information. For instance, in Figure 3.3, there won't be a path between bedroom1 and bedroom2 since there isn't a physical connection between them. Also, since the containment edges are directed from space to device, we can't have a path connecting the two spaces via a device. However, devices in bedroom1 can still be reached from bedroom2 as there exists a path (such as bedroom 2 -> livingroom -> gateway $->$ smart speaker). The enumerated paths can be considered as candidate attack paths which will be used to find vulnerable attack paths. Since we have used an adjacency list to represent the graph, the time complexity of DFS traversal is $\mathrm{O}(\mathrm{V}+\mathrm{E})$ where $\mathrm{V}$ is the number of nodes and $\mathrm{E}$ is the number of edges in the graph. Thus, the time complexity to enumerate all the paths is $\mathrm{O}\left(V^{2}\right) * \mathrm{O}(\mathrm{V}+\mathrm{E})$. The cubic time complexity of the algorithm can lead to a longer execution time for a more complex smart building layout with nodes and edges in the order of 100s. Thus, we might need to come up with some optimization strategies to reduce the number of calls to the DFS algorithm.

\subsection{Vulnerability Detection}

We identified several attack vectors to model the cyber-physical security of the smart home system. Currently, we have modeled four different cyberattacks (as presented in Table 3.1) which can be combined with a physical attack (if required). These attacks can target different layers of the Open Systems Interconnection (OSI) protocol stack. The OSI model is an ISO-standard abstract model structured into seven conceptual layers [33]. The layers form a hierarchy of functionality to receive, process, and pass information from bottom to top layer. The modeled attacks are described as follows: 
1. Light Command: For this attack, the attacker targets the MEMS (microelectromechanical systems) microphone installed in smart devices (such as Home Assistant, smart speaker, etc.) to inject inaudible and invisible laser encoded commands. This enables the attacker to inject various commands such as "set volume to 0", "change temperature to 70F", "open garage door", etc.

2. DDoS HTTP attack: HTTP Get Flood attack is one of the most common types of DDoS attacks of an application layer. During this attack, the attacker uses legitimate IP addresses which appear to be authentic, to overwhelm the webserver with multiple requests. Thus, new HTTP Get requests cannot be processed.

3. Wormhole attack: This attack involves the introduction of a malicious node to form a tunnel between a source and a destination node to misguide network traffic or modify data packets. This attack typically occurs when the two nodes are far apart and there is a need to relay the information through interim nodes.

4. Reactive jamming attack: In this attack, the attacker pretends to be a legitimate WIFI device to prevent or delay the Zigbee device's communication to the gateway. It is an optimal jamming technique since it aims at destroying only selected data packets (which are in the air) by using a short jamming signal, thus minimizing its risk of being detected.

5. Window break-in: In this attack, the attacker can break into space that is not being monitored. Thus, the attacker can gain access to space as well as the devices in that space. This is the only physical attack that we have modeled to explore the synergy between cyber and physical attacks.

We consider an attack to be successful (or a candidate path to be vulnerable) if using one or more attack vectors, the attacker can enter the house. A resultant attack path can be as 
Table 3.1: All attacks modeled in SHSim

\begin{tabular}{|c|c|c|c|c|c|}
\hline Attack vector & $\begin{array}{l}\text { Communication } \\
\text { protocol }\end{array}$ & Constraint & $\begin{array}{l}\text { Attacked } \\
\text { device }\end{array}$ & $\begin{array}{l}\text { Testing } \\
\text { device }\end{array}$ & Difficulty \\
\hline $\begin{array}{l}\text { Light command } \\
{[26]}\end{array}$ & Z- Wave,Zigbee & visibility & $\begin{array}{l}\text { Smart } \\
\text { speaker }\end{array}$ & $\begin{array}{l}\text { Smart } \\
\text { Lock }\end{array}$ & high \\
\hline $\begin{array}{l}\text { DDoS Attack } \\
\text { HTTP [12] }\end{array}$ & Z-Wave & none & Gateway & $\begin{array}{l}\text { any } \quad \text { Z- } \\
\text { Wave } \\
\text { sensor }\end{array}$ & medium \\
\hline $\begin{array}{l}\text { Wormhole } \\
\text { attack [7] }\end{array}$ & Zigbee & none & Gateway & $\begin{array}{l}\text { any zigbee } \\
\text { smart de- } \\
\text { vice }\end{array}$ & medium \\
\hline $\begin{array}{l}\text { Reactive jam- } \\
\text { ming [34] }\end{array}$ & Zigbee & none & Gateway & $\begin{array}{l}\text { any zigbee } \\
\text { smart de- } \\
\text { vice }\end{array}$ & medium \\
\hline $\begin{array}{l}\text { Window break- } \\
\text { in }\end{array}$ & $\mathrm{N} / \mathrm{A}$ & $\begin{array}{l}\text { not moni- } \\
\text { tored }\end{array}$ & NA & NA & easy \\
\hline
\end{tabular}

simple as having a single node (e.g., break-in through the window) or a much more complex attack that involves multiple nodes (e.g., performing laser-based voice command injection to unlock the door). For each resultant attack path, we estimate how difficult (low/medium/high) it is for the attacker to breach the smart home system. The implementation of this simulation is extendable to allow the addition of new attack vectors.

\subsection{Detected Attacks Visualization}

D3 is one of the most powerful tools to create data visualizations because of its flexibility and its ability to run on a web browser. It works seamlessly with existing web technologies (HTML, CSS, SVG), and can manipulate any part of the document object model [31]. We have used D3's force-directed graph to create interactive graphical visualizations of detected attacks on a smart-home environment. It uses a physics-based simulator for positioning visual 
elements. In our case, nodes are our visual elements which we position programmatically with Scalable Vector Graphics (SVG). Using forceLink(), we provide an attraction force between connected nodes such that our graph arranges itself according to our edges. Finally, we apply two more forces - "collide" and "center" - to the system. The first of these makes the elements repel each other while the second attracts the elements towards a center point. In Figure 3.4 we can see an example of the output visualization. The attack path is highlighted using a curved black line wherein the attacker targets the smart speaker (highlighted with red outline) to unlock the smart lock. We can also see the type of attack used along with the attack difficulty level. We provide a list of all resultant attack paths for the user to select to get the corresponding visualization.

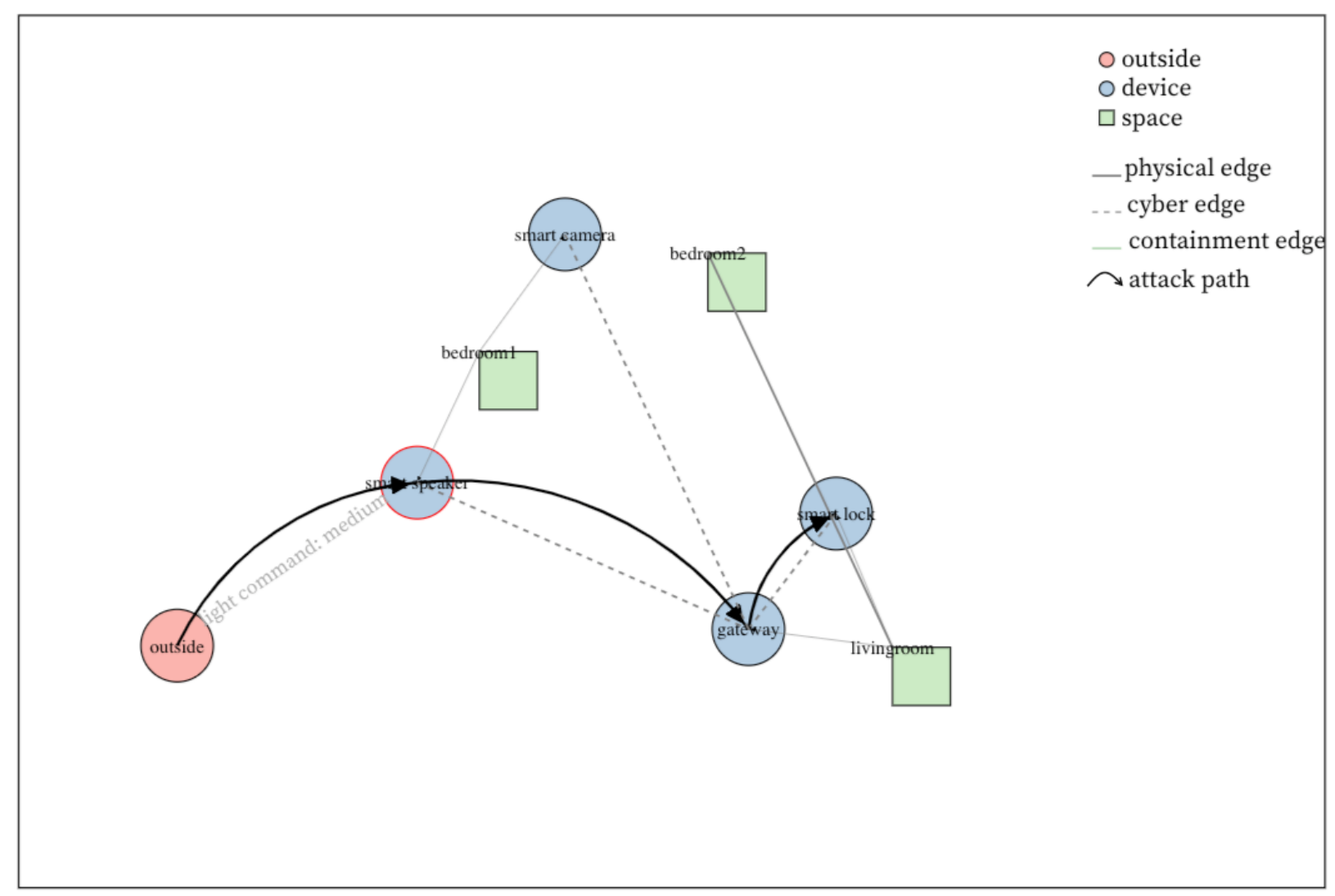

Figure 3.4: Light command attack on Smart home layout 1 


\section{Chapter 4}

\section{Results}

We performed a few experiments on three different smart home designs to cover divergent scenarios and test the validity of our simulation engine.

\subsection{Smart home layout 1}

As shown in Figure 4.1, we defined a smart home layout with 3 spaces (bedroom1, bedroom2,

and livingroom) and 4 smart home devices. Bedroom1 (or the Master bedroom) contains a smart speaker which can also act as a voice-enabled assistant (e.g., Google home or Echo dot) and a smart camera that monitors the space. Bedroom2 doesn't contain any smart device. The living room has a gateway that connects to other smart home devices and relays data packets. The living room also has a smart lock installed at the main door. In Figure 3.3 we can see the graph generated for the smart home layout. On running the simulation, we get a list of attack paths with relevant information such as attack difficulty level, attack type, targeted node, etc.

\subsubsection{Experiment 1a: Space monitored vs not monitored}

For the given smart home layout, we experimented by first adding the smart camera to monitor bedroom 1 and then removing it to observe the differences in output. We identified 


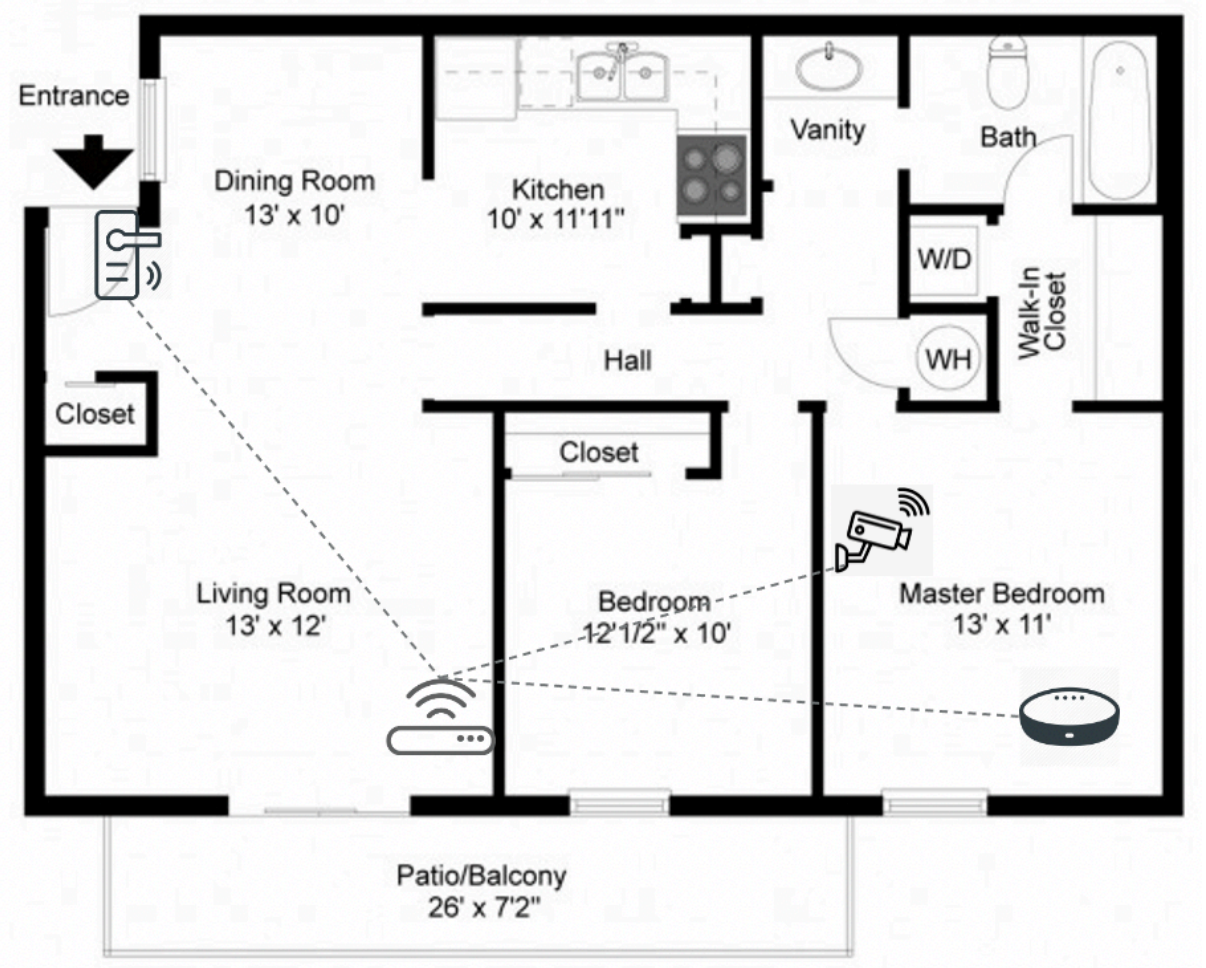

Figure 4.1: Smart home layout 1

9 attack paths when the space was being monitored and 12 attack paths when it was not being monitored. The difference lies in the fact that when space is not being monitored, the attacker can perform a physical attack on bedroom1 window and enter the space. In Figure 3.4, we can see one of the resultant paths wherein the attacker uses the Light command [26] attack to target the smart speaker and unlocks the smart lock. The engine estimates that this attack has a "medium" difficulty level based on the attack model. Also, we eliminate some of the unknown parameters such as time taken to hack the passcode of the smart lock since it depends entirely on the kind of smart lock installed. We found that certain smart locks such as Nest X Yale locks are much more secure than August smart locks. Other possible attacks include a DDoS HTTP [12] attack on the gateway which blocks its internet connectivity, thus restricting the homeowner from using commands which involve an internet connection. 


\subsubsection{Experiment 1b: Smart Speaker visibility}

For the second part of our experiment, we removed the visibility of the smart speaker from the outside and ran the simulation again. We found out that the attacker was no longer able to target the smart speaker from outside since it was not in the line of sight. However, since bedroom1 was not being monitored, the attacker was able to physically enter and then perform the Light command attack, as now the smart speaker was in the line of sight.

\subsection{Smart home layout 2}

The second smart home layout is much more complex. We took the floor plan of a real-life duplex house with smart-home security and an automation system. In Figure 4.2, we can see the floor plan of the smart home which consists of a living room, kitchen \& dining, hall, and 3 bedrooms. Figure 4.3 describes the internet connectivity setting wherein we have a WIFI modem placed in the living room providing connectivity to the smart-home security system, the smart-home automation system, and other devices. In Figure 4.4, we can see the placement of smart-security-related devices such as smart cameras, smart doorbells, motion sensors, etc. The first base station placed in the living room connects to the WIFI and communicates to all of the smart-security devices on a common network (i.e., Z-Wave). Similarly, in Figure 4.5 we can see the placements of smart-automation devices such as home assistants, smart thermostats, etc. The second base station connects to the internet and communicates with all smart home devices over the Zigbee network. 

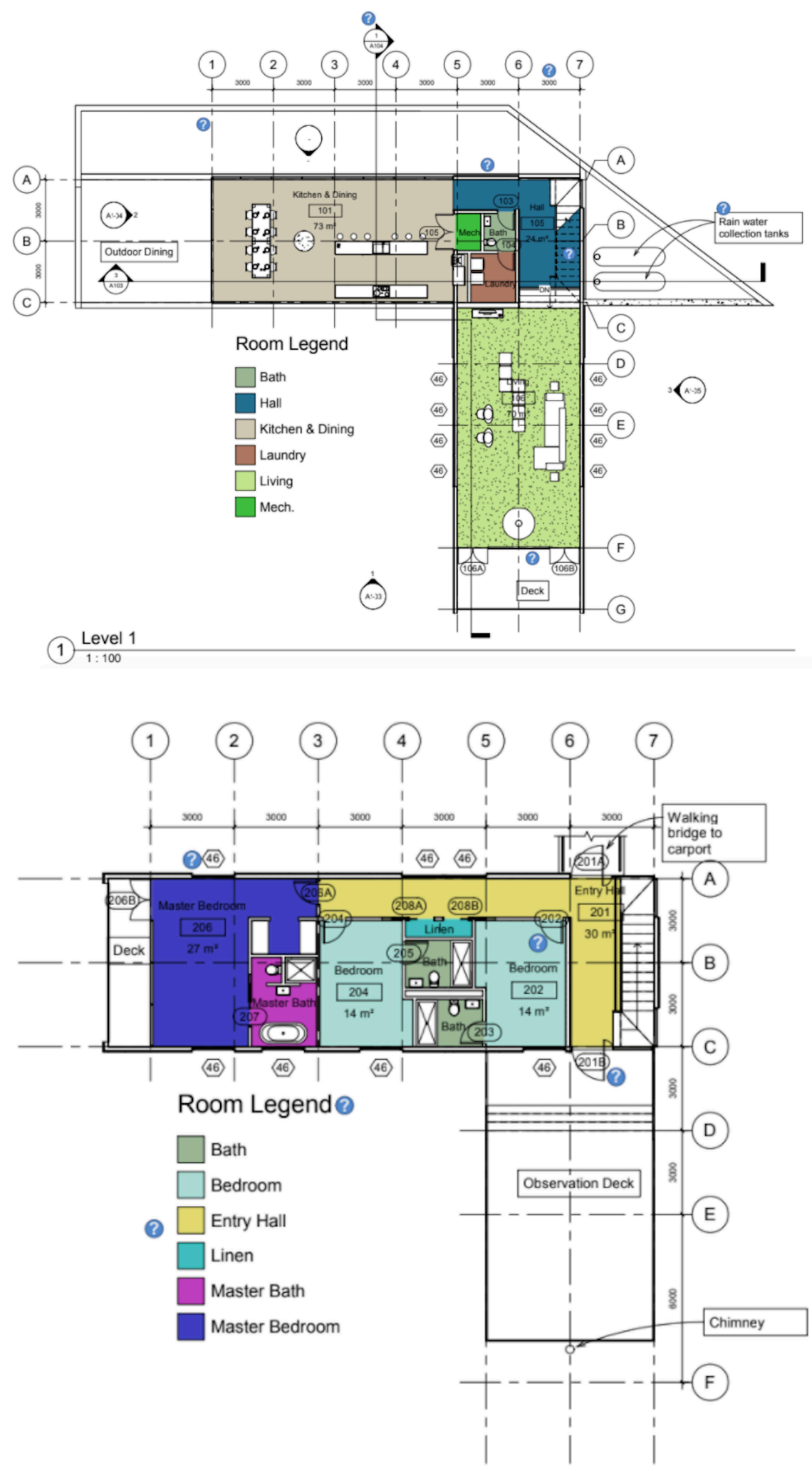

(2) Level 2

Figure 4.2: Floor plan of Smart home layout 2 [1] 


\section{The home Internet setting}

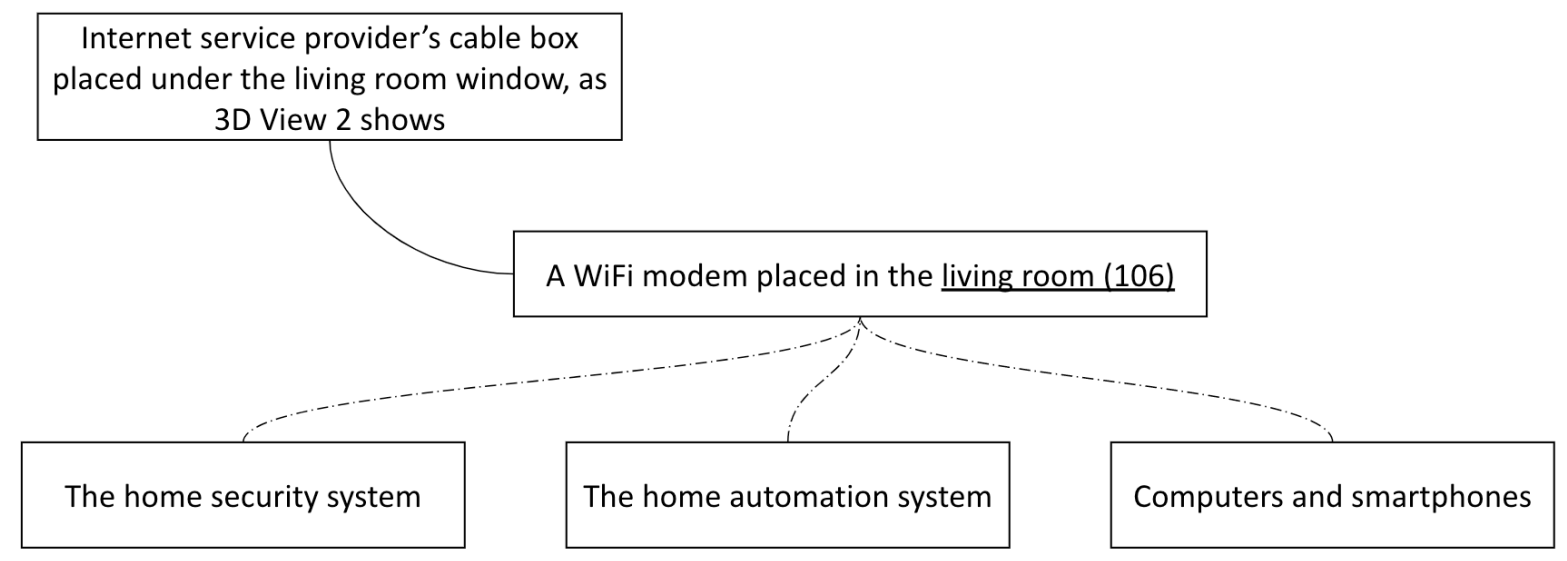

Legend:

Physical cable connection

WiFi connection

Figure 4.3: Internet setting in Smart home layout 2

\subsubsection{Experiment 2: All spaces monitored}

For this experiment we observed 21 different attack paths on running the simulation. Since all of the spaces were monitored, the attacker couldn't perform any physical attack. We can divide 21 different attack paths into 3 cyber-physical attacks:

1. DDoS HTTP attack: Since Z-Wave devices are vulnerable to DDoS attacks, Gateway 1 has been targeted to block its communication with other smart-security devices such as entry sensors, smart cameras, etc. In Figure 4.6, we can see one such attack path which blocks the data transmission between the Gateway 1 and Entry sensor 2.

2. Wormhole attack: Since Zigbee devices are vulnerable to Wormhole attack [7], Gateway 2 has been targeted to misguide the network traffic between the gateway and other Zigbee devices such as home assistants, smart thermostats, etc. 
The home security system setting

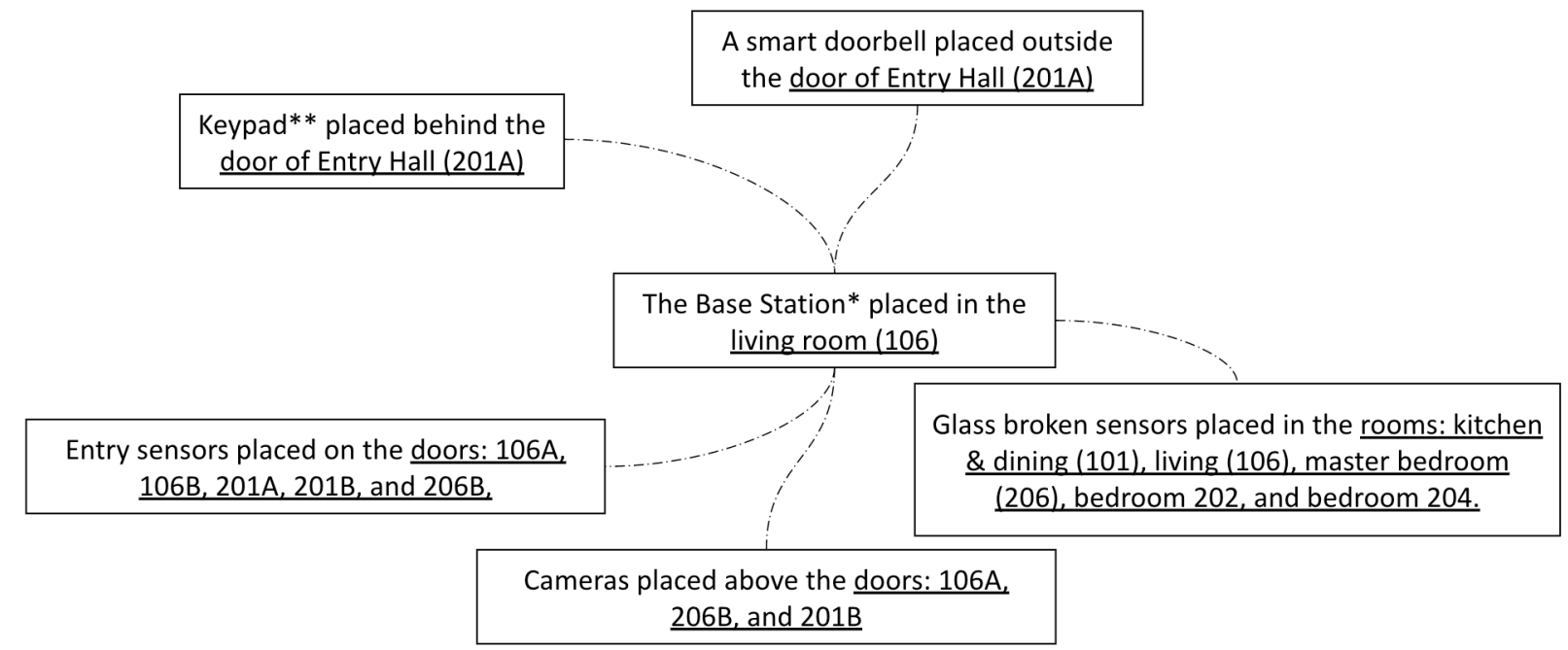

*The Base Station is the central device that communicates with the call center, connects to WiFi and all devices, and sends audio alarms.

** The keypad is where password can be input to disarm the system.

Figure 4.4: Security system setting of Smart home layout 2

The home automation system setting

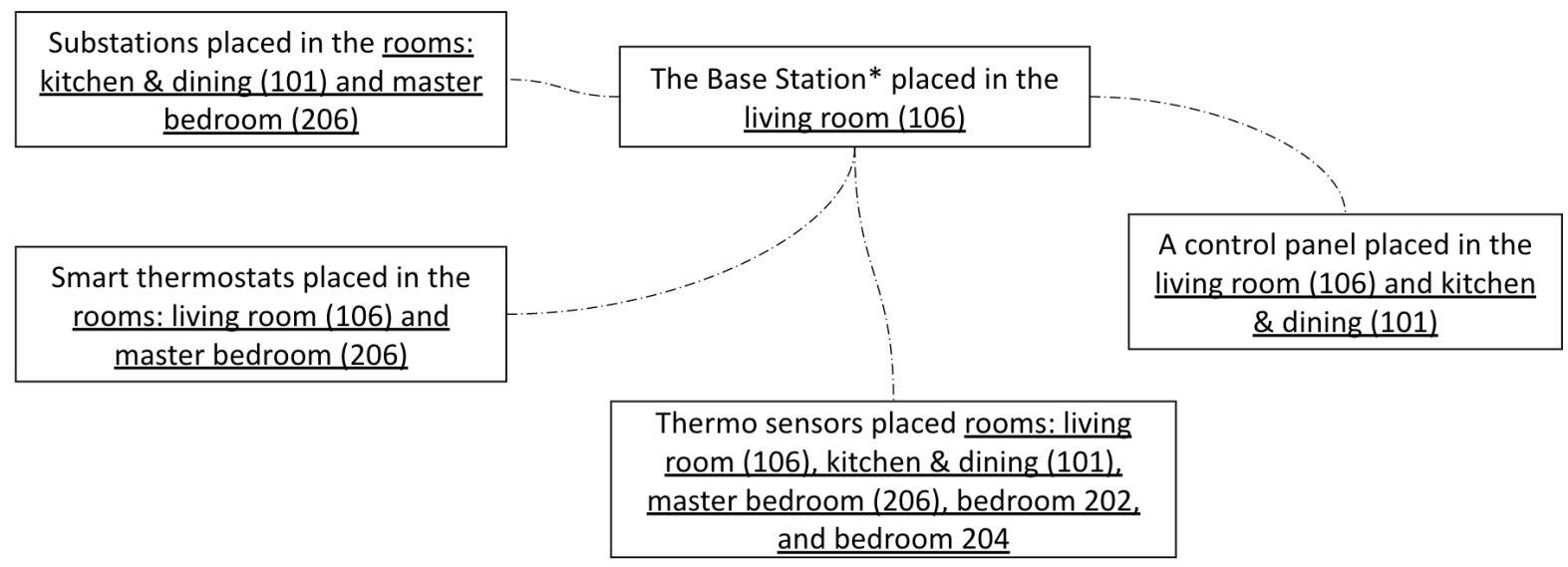

*The examples of the smart home base station can be Google Home, Amazon Echo, etc. They have voice control functions, connects to the Internet, and communicates with all smart home devices

** The examples of the substation can be Google Home mini, Echo dot, etc.

Figure 4.5: Automation system setting of Smart home layout 2 


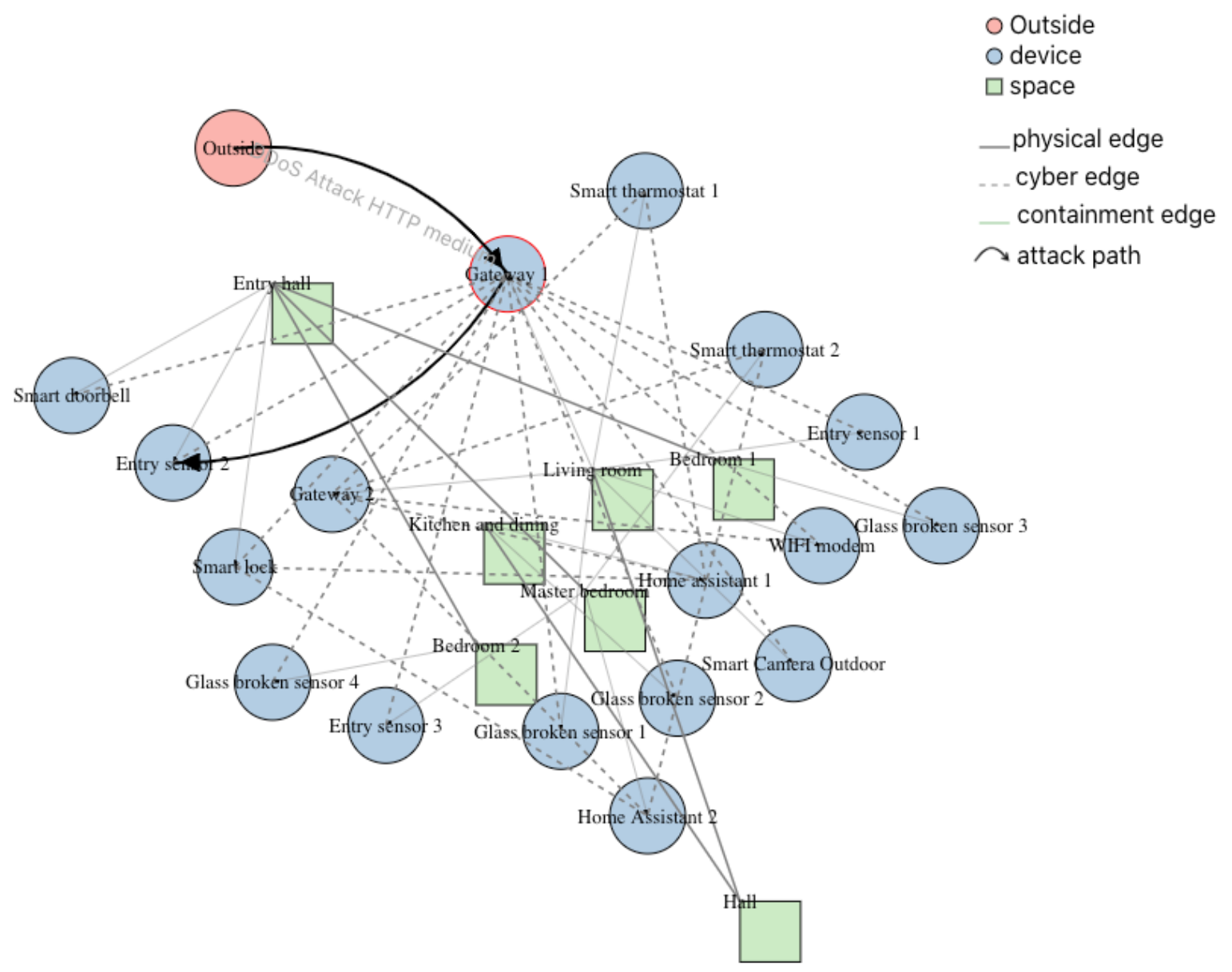

Figure 4.6: An example DDoS attack on smart security system of Smart home layout 2

3. Light command attack: In Figure 4.7, we can see the attacker targets the home assistant devices which are visible from outside to unlock the smart lock. The attacker exploits the vulnerability of MEMS microphones to remotely inject inaudible and invisible commands into voice assistants.

\subsection{Smart home layout 3}

We took the design inspiration of the third smart home layout from Surreal System's website [27]. On their homepage, they provide the floor plan (as shown in Figure 4.8) of a smart 


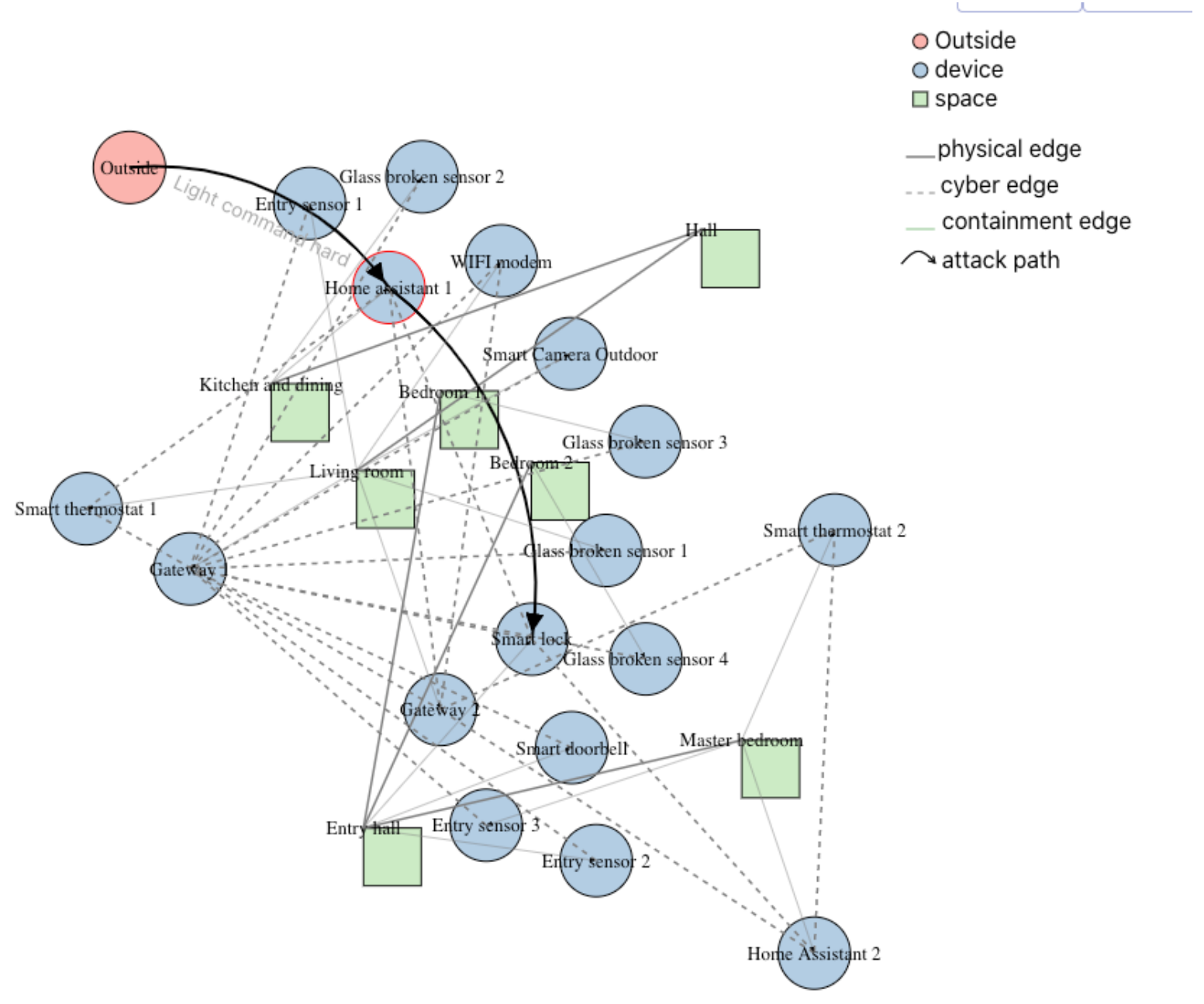

Figure 4.7: Light command attack on Smart home layout 2

home which consists of a living room, bedroom, storage room, and a garage. The living room has a voice-enabled assistant and a smart lighting system. The bedroom and living room windows have motorized smart shades. The storage room contains the gateway and the garage is being monitored via a smart camera. The devices communicate with others over the Zigbee network and can be controlled via a switch, mobile app, or home assistant. 


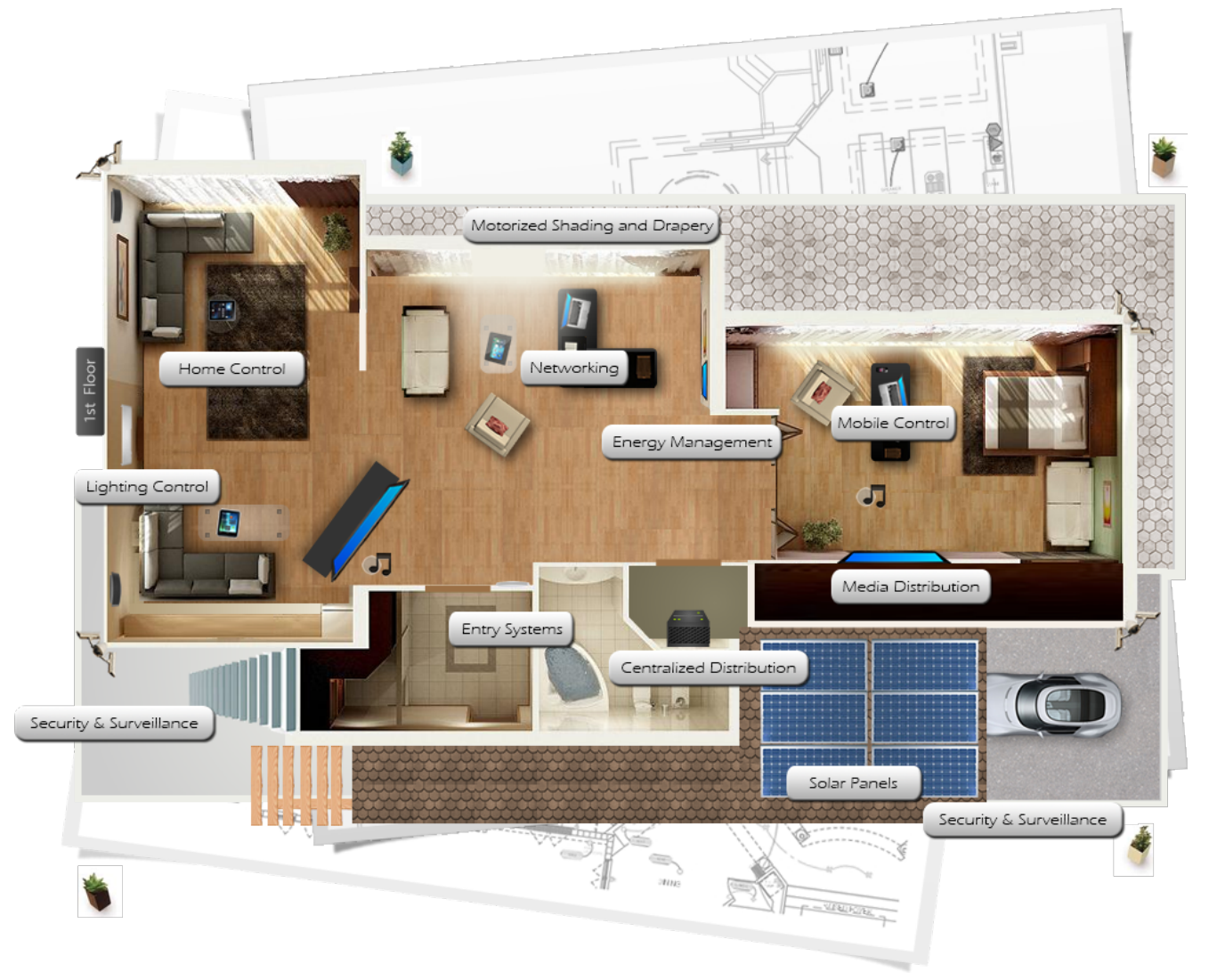

Figure 4.8: Smart home layout 3

\subsubsection{Experiment 3: No occupants}

For this experiment, we configured the spaces with no occupants while running the simulation. We found the smart home design being quite vulnerable as the simulation provided us 31 different attack paths. The attacker was able to exploit the vulnerability of the Zigbee network and perform three different kinds of cyber-physical attacks:

1. Wormhole attack: The attacker inserts a malicious node to form a tunnel between the Home assistant and other smart devices such as smart light, smart shades, etc. By doing this, the attacker can misguide network traffic and even modify data packets. The attacker has the option of doing the entire process from outside the home (if he/she can receive a signal) or by entering the house (since most of the spaces are not 
being monitored or occupied.)

2. Concealed jamming attack [3]: In this attack method, the attacker targets the gateway to jam or delay the data transmission between gateway and other smart devices such as Home assistant, smart lights, smart shades, smart camera, etc. In Figure 4.9, we can see one such attack where the attacker jams the network between smart shades and the gateway. Thus, if the resident wants to operate the smart shades using the Home assistant, they would fail. However, they can still operate it via a switch or mobile app.

3. Light command attack: The attacker targets the Home assistant device present in the bedroom to inject laser commands. Thus, the attacker can gain control over other controllable devices such as smart shades and smart lights.

4. Physical attack: The attacker has the option to break into the house through the living room and bedroom windows as the spaces are not being monitored or occupied. The attacker cannot enter through the garage as it is being monitored by a camera.

From this experiment, it's evident that not having a smart home security system can make it easy for the attacker to perform cyber-physical attacks when no one is at home.

The experiments conducted illustrate that the current generation of smart home systems are not always sufficiently secure. The attacker, by sensing weak entry points, can launch cyber-physical attacks to either enter the house or gain access to the network. We found that the Base stations or the gateways are the prime targets to various cyber attacks. Spaces which are not monitored or occupied can lead to physical attacks. The attacker on entering the house can sense neighbouring spaces and plan his next attack accordingly. 


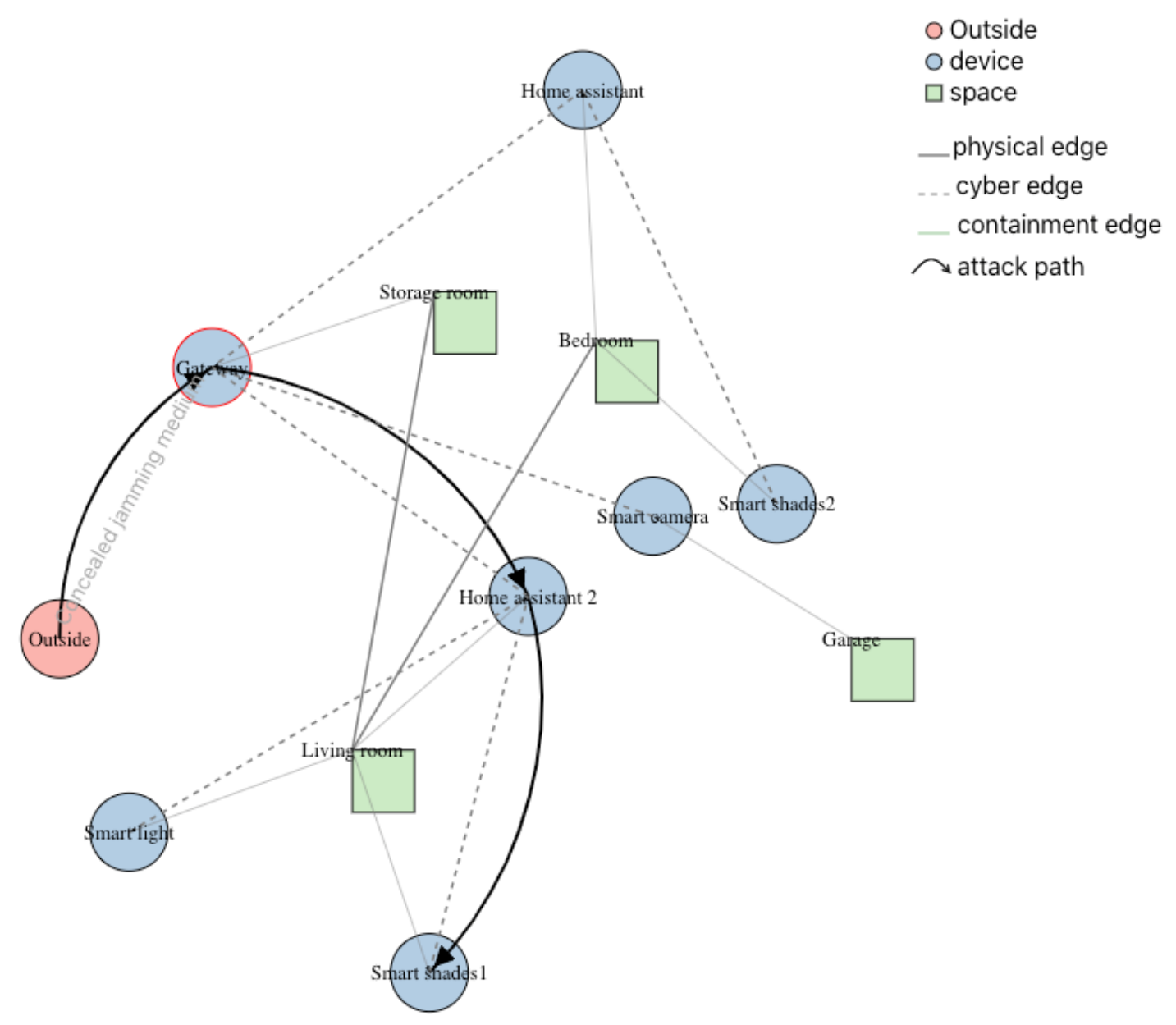

Figure 4.9: Concealed jamming attack on Smart home layout 3 


\section{Chapter 5}

\section{Discussion}

SHSim can be used by researchers and customers to analyze the security of their smart home systems. Using the simulation engine, device manufacturers can find loopholes and try to fix them. Typically, a smart homeowner installs devices that are compatible with one common smart home solution (e.g., Google, Amazon, etc.) to avoid having multiple different gateways. However, our simulation engine can handle multiple smart home solution ecosystems.

Even though we conducted several experiments to test the simulation engine and explore different attack paths, more study is needed to further improve the proposed system.

1. More experiments using a real-life smart home setting can bring out interesting insights as well as test the robustness of the simulation engine to handle different circumstances.

2. Currently, our attack models are based on the vulnerability of Z-Wave and Zigbee communication protocols. However, there exist many more communication protocols such as Insteon, Thread, KNX, BACnet, etc. for building a smart-home network which need to be investigated.

3. We made certain assumptions for successfully running the simulation engine which can be taken as parameters in a future version of SHSim. For instance, we assume that the attacker from outside gets the network signal to access a few smart home devices. However, we do not consider signal information in our modeling. Thus, for future 
development, we can build a large database of smart home devices with their profile and provide a user the option to select a device from the list.

4. In our attack model, we assume that all of the smart devices that work on a vulnerable communication protocol are vulnerable devices. However, it might not always be the case. Smart home vendors can push firmware updates on top of communication protocols to fix certain vulnerabilities. Thus, in future iterations, one can focus on device-level vulnerability rather than network-level vulnerability.

5. Currently we take user input about building layout and device placements through HTML form elements. However existing work shows that the corresponding graph for spatial breach analysis can be built using the building's BIM information [20]. Thus, in a future version of SHSıM, we can use a similar methodology to extract smart building information from BIM. Also, current smart home services already collect some spatial location data of the devices to provide better service, so one can explore the idea of extracting such location data for device placement. 


\section{Chapter 6}

\section{Conclusions}

Cyber-physical security for smart-building is a relatively new topic and requires a scientific tool to help study potential cyber-physical attacks and impacts. This research has proposed a system that can simulate cyber-physical systems using graphs, and offered a prototype that is expandable and capable of analyzing the threat to the smart-home environment. We conducted several experiments to analyze the vulnerability of different smart home designs. We found that gateways or hubs are primary targets of cyberattacks. Also, the lack of a smart security system can provide an opportunity for a physical attack which in turn can result in cyberattacks on smart devices. Future directions of improvements include more detailed modeling that better reflects reality, optimized algorithms for simulation, different

algorithms to simulate a different aspect of the system, a standardized attack and impact library, and standardized modeling of devices and protocols. 


\section{Bibliography}

[1] Autodesk. Sample Revit project, 2021. URL https://www.autodesk. com/products/ revit/overview?term=1-YEAR. [Accessed April 21st, 2021].

[2] Mike Bostock. D3.js - Data-Driven Documents, 2012. URL http://d3js.org/. [Accessed March 21, 2021].

[3] Zicheng Chi, Yan Li, Xin Liu, Wei Wang, Yao Yao, Ting Zhu, and Yanchao Zhang. Countering Cross-Technology Jamming Attack. In Proceedings of the 13th ACM Conference on Security and Privacy in Wireless and Mobile Networks, WiSec '20, page 99-110, New York, NY, USA, 2020. Association for Computing Machinery. ISBN 9781450380065. doi: 10.1145/3395351.3399367. URL https://doi.org/10.1145/ 3395351.3399367. Accessed 9-February-2021].

[4] Pierre Ciholas, Aidan Lennie, Parvin Sadigova, and J. Such. The Security of Smart Buildings: a Systematic Literature Review. ArXiv, abs/1901.05837, 2019.

[5] Michela Cozza, Antonella De Angeli, and Linda Tonolli. Ubiquitous technologies for older people. Personal and Ubiquitous Computing, 21, 06 2017. doi: 10.1007/ s00779-017-1003-7.

[6] Behrang Fouladi and Sahand Ghanoun. Honey, I'm Home!!, Hacking Z-Wave Home Automation Systems. In Black Hat, 2013. URL https://cybergibbons.com/ wp-content/uploads/2014/11/honeyimhome-131001042426-phpapp01.pdf.] [Accessed March 17th, 2021].

[7] Julian Ramirez Gómez, Héctor Fernando Vargas Montoya, and Alvaro Leon Henao. 
Implementation of a Wormhole Attack on Wireless Sensor Networks with XBee S2C Devices. In Jairo E. Serrano C. and Juan Carlos Martínez-Santos, editors, Advances in Computing, pages 98-112, Cham, 2018. Springer International Publishing. ISBN 978-3-319-98998-3.

[8] Miguel Grinberg. Flask web development: developing web applications with Python. O’Reilly Media, Inc., 2018.

[9] M. Hager, S. Schellenberg, J. Seitz, S. Mann, and G. Schorcht. Secure and QoS-aware communications for smart home services. In 2012 35th International Conference on Telecommunications and Signal Processing (TSP), pages 11-17, 2012. doi: 10.1109/ TSP.2012.6256188.

[10] Ryan Heartfield, George Loukas, Sanja Budimir, Anatolij Bezemskij, Johnny R.J. Fontaine, Avgoustinos Filippoupolitis, and Etienne Roesch. A taxonomy of cyberphysical threats and impact in the smart home. Computers Security, 78:398428, 2018. ISSN 0167-4048. doi: https://doi.org/10.1016/j.cose.2018.07.011. URL https://www.sciencedirect.com/science/article/pii/s0167404818304875. [Accessed 2nd February, 2021].

[11] Y. Hu, Dominique Tilke, Taylor Adams, Aaron S. Crandall, D. Cook, and M. SchmitterEdgecombe. Smart home in a box: usability study for a large scale self-installation of smart home technologies. Journal of Reliable Intelligent Environments, 2:93-106, 2016.

[12] Ladislav Huraj, Marek Šimon, and Tibor Horák. Resistance of IoT Sensors against DDoS Attack in Smart Home Environment. Sensors, 20(18), 2020. ISSN 1424-8220. URL https : //www.mdpi .com/1424-8220/20/18/5298. [Accessed 2nd February, 2021].

[13] Prem Khatri. Why some developers prefer Node.js React.js for application de- 
velopment, 2021. URL https://www.chetu.com/blogs/technical-perspectives/ nodejs-and-reactjs-for-app-development.php. [Accessed April 22nd, 2021].

[14] Soo-Cheol Kim, Young-Sik Jeong, and Sang-Oh Park. RFID-Based Indoor Location Tracking to Ensure the Safety of the Elderly in Smart Home Environments. Personal Ubiquitous Comput., 17(8):1699-1707, December 2013. ISSN 1617-4909. doi: 10.1007/ s00779-012-0604-4. URL https://doi .org/10.1007/s00779-012-0604-4.

[15] Claire Lloyd. How to Design a Smart Home: An Expert Guide, 2020. URL https: //www.homebuilding.co.uk/advice/how-to-design-a-smart-home. [Accessed April 19th, 2021].

[16] Sumit Majumder, Emad. Aghayi, Moein Noferesti, Hamidreza Memarzadeh-Tehran, Tapas Mondal, Zhibo Pang, and M. Jamal Deen. Smart Homes for Elderly HealthcareRecent Advances and Research Challenges. Sensors, 17(11), 2017. ISSN 1424-8220. doi: 10.3390/s17112496. URL https://www .mdpi.com/1424-8220/17/11/2496. [Accessed 1st April-2021].

[17] Material-UI. React Paper Component, 2021. URL https://material-ui.com/ components/paper/. [Accessed April 21st, 2021].

[18] T. Mundt and P. Wickboldt. Security in building automation systems - a first analysis. In 2016 International Conference On Cyber Security And Protection Of Digital Services (Cyber Security), pages 1-8, 2016. doi: 10.1109/CyberSecPODS.2016.7502336.

[19] Node.js. About Node.js. URL https://nodejs.org/en/about/. [Accessed April 21st, 2021].

[20] Stuart Porter, Terence Tan, Tele Tan, and Geoff West. Breaking into BIM: Performing static and dynamic security analysis with the aid of BIM. Automation in Construction, 
40:84-95, 2014. ISSN 0926-5805. doi: https://doi.org/10.1016/j.autcon.2013.12.002. URL https://www.sciencedirect.com/science/article/pii/S0926580513002148. [Online; accessed 7-April-2021].

[21] François Portet, Michel Vacher, Caroline Golanski, Camille Roux, and Brigitte Meillon. Design and evaluation of a smart home voice interface for the elderly Acceptability and objection aspects. Personal and Ubiquitous Computing, 17:1-18, 01 2012. doi: 10.1007/s00779-011-0470-5.

[22] React. About React.js, 2021. URL https://reactjs.org/. [Accessed April 20th, 2021].

[23] Rosslin Robles and Tai-hoon Kim. Applications, Systems and Methods in Smart Home Technology: A Review. 15, 012010.

[24] S. Safaric and K. Malaric. Zigbee wireless standard. In Proceedings ELMAR 2006, pages 259-262, 2006. doi: 10.1109/ELMAR.2006.329562.

[25] Nimalaprakasan Skandhakumar, Farzad Salim, Jason Reid, Robin Drogemuller, and Ed Dawson. Graph theory based representation of building information models for access control applications. Automation in Construction, 68:44-51, 08 2016. doi: 10. 1016/j.autcon.2016.04.001.

[26] Takeshi Sugawara, Benjamin Cyr, Sara Rampazzi, Daniel Genkin, and Kevin Fu. Light Commands: Laser-Based Audio Injection Attacks on Voice-Controllable Systems. In 29th USENIX Security Symposium (USENIX Security 20), pages 26312648. USENIX Association, August 2020. ISBN 978-1-939133-17-5. URL https: //www. usenix.org/conference/usenixsecurity20/presentation/sugawara. [Accessed 17th April, 2021]. 
[27] Surreal System. Smart Home Layout, 2021. URL https://www. surrealsystems .com/ solutions/home-solutions/smart-home-layout. [Accessed April 18, 2021].

[28] TaraSeals. BlackHat: Critical ZigBee Flaw Compromises Smart Homes, 2015. URL https://www.infosecurity-magazine.com/news/ blackhatcritical-zigbee-flaw-smart/. [Accessed March 17th, 2021].

[29] Katell Thielemann. Organizations now facing new threats - Protecting cyberphysical systems, Dec. 22, 2020 . URL https://www.itproportal.com/features/ organizations-now-facing-new-threats-protecting-cyber-physical-systems/. [Online; accessed 9-April-2021].

[30] R.J. Trudeau. Introduction to Graph Theory. Dover Books on Mathematics. Dover Pub., 1993. ISBN 9780486678702. URL https://books.google.com/books?id= 8nYH50YEW24C. [Online; accessed 9-April-2021].

[31] Wikipedia contributors. Document object model - Wikipedia, the free encyclopedia, 2021. URL https://en.wikipedia.org/w/index.php?title=Document_Object_ Model\&oldid=1014012523. [Online; accessed 9-April-2021].

[32] Wikipedia contributors. Z-wave - Wikipedia, the free encyclopedia, 2021. URL https://en.wikipedia.org/w/index.php?title=Z-Wave\&oldid=1000389478. [Online; accessed 9-April-2021].

[33] Wikipedia contributors. OSI protocols - Wikipedia, The Free Encyclopedia, 2021. URL https://en.wikipedia.org/w/index.php?title=0SI_protocols\& oldid=1010987681. [Online; accessed 20-May-2021].

[34] Matthias Wilhelm, Ivan Martinovic, Jens Schmitt, and Vincent Lenders. Short Paper: 
Reactive Jamming in Wireless Networks-How Realistic is the Threat? pages 47-52, 06 2011. doi: 10.1145/1998412.1998422.

[35] Tatsuya Yamazaki. The ubiquitous home. International Journal of Smart Home, 1, 02 2007.

[36] S. T. Zargar, J. Joshi, and D. Tipper. A Survey of Defense Mechanisms Against Distributed Denial of Service (DDoS) Flooding Attacks. IEEE Communications Surveys Tutorials, 15(4):2046-2069, 2013. doi: 10.1109/SURV.2013.031413.00127. 